\title{
Can psychedelic compounds play a part in drug dependence therapy?
}

\author{
Ben Sessa and Matthew W. Johnson
}

\section{Summary}

After a 40-year hiatus there is now a revisiting of psychedelic drug therapy throughout psychiatry, with studies examining the drugs psilocybin, ketamine, ibogaine and ayahuasca in the treatment of drug dependence. Limitations to these therapies are both clinical and legal, but the possibility of improving outcomes for patients with substance dependency imposes an obligation to research this area.

\section{Declaration of interest}

None.
Ben Sessa (pictured) is a consultant psychiatrist in substance misuse working for 'AddAction' in Weston-Super-Mare, North Somerset, and a senior research fellow at Cardiff University Medical School, UK. Matthew W. Johnson is Associate Professor at Johns Hopkins School of Medicine, Baltimore, USA

\section{'The only radical remedy I know for Dipsomania is Religiomania'}

William James in The Varieties of Religious Experience

After one hundred years of modern psychiatry the treatments available for enduring remission from drug dependence for alcohol, opiates, stimulants and nicotine remain poor. Although pharmacological treatments exist they are associated with substantial failure rates. A wealth of studies explored psychedelic drug-assisted therapy in the 1950s and 1960s, but this research was curtailed prematurely in the wake of the 1960s recreational drug phenomenon. After a hiatus of several decades this research is being revisited with a number of contemporary studies examining the drugs psilocybin, ketamine, ibogaine and ayahuasca to directly tackle drug dependence.

\section{Why concentrate on drug dependence?}

People who are drug-dependent are often stigmatised, maligned by society and blamed. However, the experience of the authors - a child psychiatrist who now works in adult substance misuse (B.S.) and a behavioural psychologist who studies the environmental determinants of drug use (M.W.J.) - is that many of these patients are helpless, needy victims of adverse psychosocial circumstances. Their trajectory into drug dependence has been a journey from childhood trauma. Now they face not only their unresolved trauma but also the plight of drug dependence that ties them to a lifestyle of psychosocial and financial dysfunction. Because of the complexity of their aetiology and the psychological and physiological dependence that results, these disorders are very difficult to treat.

\section{Alcohol addiction}

One adult in 20 in the UK is a dependent drinker and one quarter of all adults drink in a hazardous fashion. ${ }^{2}$ Alcohol dependency and misuse is a major factor in offending behaviour. It is strongly related to crime, including domestic abuse, antisocial behaviour, public disorder, sexual assault and motoring offences. ${ }^{3}$ A metaanalysis of 361 controlled studies of treatments for alcohol dependence in 2002 identified 46 possible interventions. ${ }^{4}$ The brief intervention approach ranked highest and motivational enhancement ranked second. Pharmacotherapy with the gamma-aminobutyric acid (GABA) agonist acamprosate and the opiate antagonist naltrexone ranked third and fourth respectively. The lowest ranked approaches were designed to educate, confront, shock or foster insight regarding the nature of alcoholism. There remains a lack of coherence and agreement about the most efficacious alcohol dependence treatment. Meanwhile, taking into account alcohol-related health disorders and disease, crime and antisocial behaviour, accidents, loss of productivity in the workplace and domestic problems, the Department of Health estimates alcohol misuse is now costing around $£ 20$ billion a year in England alone. ${ }^{5}$

\section{Opiate dependence}

Misuse of heroin and related opioids is a major public health concern, with over 123000 people injecting heroin in the UK. Those who use heroin have a 12 -fold increase in mortality relative to the general population ${ }^{6}$ and often present with severe physical, mental, social and criminal legal complications. Methadone and buprenorphine are the most commonly prescribed medications for opioid dependence. They have consistently been found to increase treatment retention and decrease opioid misuse, mortality, HIV risk and crime. However, these substitution treatments have limitations. Methadone is associated with medical risk and is contraindicated for some patients, and both medications are dangerous in combination with sedative drugs. ${ }^{7}$

\section{Stimulant dependence}

Cocaine is the second most popularly used illegal drug in the UK. In its powder form it may be snorted or injected, and in its freebase form crack cocaine can be smoked and injected. There is no accepted substitution treatment for cocaine or other stimulants as there are for opiates. Treatments rather involve psychological strategies including contingency management, cognitive-behavioural therapy and motivational interviewing. Drug therapies are largely symptomatic (i.e. addressing comorbid depression, anxiety and insomnia) or treat coexisting opiate dependence.

\section{Nicotine dependence}

Although people who smoke are less marginalised than those using other drugs, tobacco is associated with more deaths than any other legal or illegal drug. The numbers are staggering - 
tobacco kills over 1.2 million people annually in Europe alone. ${ }^{8}$ Although the most effective medications (varenicline, bupropion, nicotine replacement) improve success rates, the large majority of patients relapse even with these medications. ${ }^{9}$ There is a desperate need for improvement. An ongoing open-label pilot study in the laboratory of one author (M.W.J.) is showing promising results using psilocybin combined with cognitive-behavioural therapy for smoking cessation. If efficacy and safety are supported by a randomised trial, this approach may hold potential to have a substantial public health impact given the shocking mortality caused by smoking.

\section{Psychedelic therapy and substance misuse}

In the 1950s and 1960s psychiatrists Humphry Osmond and Abram Hoffer at the Weyburn Mental Hospital, Saskatchewan, Canada, used lysergic acid diethylamide (LSD) to provide a clinician-induced organic psychosis to encourage sobriety. They found that it was mystical spiritual experiences - not psychotic experiences - with the drugs that were associated with treatment success. With LSD they reported abstinence rates far surpassing other treatments before or since. Osmond, who famously coined the term 'psychedelic', also administered LSD to Bill Wilson, the founder of Alcoholics Anonymous, who recognised LSD therapy as beneficial for alcohol dependence. A large number of studies to treat alcohol dependence with LSD psychotherapy were conducted by other researchers in the 1960s until psychedelic research collapsed for sociopolitical reasons in the wake of largescale recreational drug use. ${ }^{10}$

\section{Cross-cultural use of psychedelics}

There are examples of the naturalistic use of psychedelic plants to tackle addictions by indigenous populations: these include the West African use of the iboga root (containing ibogaine), the South American use of ayahuasca (containing dimethyltryptamine) and the North American use of the peyote cactus (containing mescaline); all of which have been reported to reduce rates of alcohol dependence.

\section{Possible therapeutic mechanisms}

Personality change is relevant to drug dependence, given that maladaptive personality traits often accompany drug use disorders. Two observational studies from the early 1960s suggested positive personality and other therapeutic changes in criminal offenders. ${ }^{11}$ With colleagues, M.W.J. has recently revisited prisoner personality change interventions with positive results. ${ }^{12}$ This idea of mystical-spiritual experience resulting in personality change was also explored recently in studies in which volunteers rated the psilocybin experience as having substantial personal meaning and spiritual significance. Subsequently sustained positive changes in attitudes and behaviour were consistent with changes rated by community observers. ${ }^{13}$ Another possible mechanism for the anti-addictive properties of hallucinogens may involve an 'afterglow' period of several weeks described in early clinical research. It was suggested that this period gave patients emotional strength to continue abstinence and experience decreased cravings. ${ }^{14}$ A recent paper by Bogenschutz \& Pommy further explored potential psychological and biological therapeutic mechanisms of psychedelics in the treatment of substance misuse disorders. ${ }^{15}$

\section{Contemporary studies}

A team in Russia in the 1990s, driven by the theory behind Osmond's 1950s studies, investigated the potential role for psychedelic drug-assisted psychotherapy with ketamine for both alcohol and opiate addictions. The results of subsequent placebo-controlled studies on more than 1000 patients with alcoholism showed that ketamine produced total abstinence for more than a year in $66 \%$ of those in the ketamine group, compared with just $24 \%$ of the control group. ${ }^{16}$ Despite positive published results the Russian Federation forbids further research with ketamine. In addition to the previously mentioned study of psilocybin and nicotine addiction, an open-label pilot study investigating psilocybin-assisted psychotherapy in alcohol dependence is under way at the University of New Mexico (clinicaltrials.gov registration number NCT01534494). There are also observational studies in Mexico and New Zealand of ibogaine-assisted therapy and a study conducted in Canada looking at the role of ayahuasca in the treatment of drug dependence (see http://maps.org for details of all these studies).

\section{Theoretical objections to psychedelic treatment}

There may be objections to treating drug dependence with other potentially misused drugs. However, current treatments for drug dependence already involve maintenance pharmacotherapy with controlled drugs. In seeking enduring remission, psychedelic drug-assisted psychotherapy is not simply maintenance therapy. Moreover, although psychedelic drugs can be misused, many of them (psilocybin, LSD) do not support compulsive drug-seeking.

\section{The psychedelic renaissance}

After a 40-year hiatus there is now a revisiting of psychedelic drug therapy throughout psychiatry. ${ }^{17}$ Research teams at major academic institutions worldwide are investigating psychedelic drug-assisted therapy and several independent research groups have emerged to work collaboratively on the cohesive organisation of research. This research is still in its early stages and the most effective methods have yet to be evaluated. There are several limitations. For example, psychotherapy sessions that last for many hours would be expensive and are unlikely to be a first-line intervention. Moreover, some individuals would be excluded from psychedelic therapy for safety reasons. Another challenge is that many of these drugs are restricted at the Schedule I or Class A level, forbidding all medical use outside of highly regulated medical research. A medical future for these compounds would require a change in regulatory status (e.g. to the level at which compounds with accepted medical use such as morphine and amphetamine are regulated) and a plan for the manufacture of approved compounds.

Patients with drug dependence deserve the opportunity for the best available treatments from their psychiatric services. If there is a chance that psychedelic drug-assisted psychotherapy could improve outcomes for this population of patients, we owe it to them to research this area.

Ben Sessa, MBBS, MRCPsych, AddAction, Weston-Super-Mare, Cardiff University Medical School, UK; Matthew W. Johnson, PhD, Johns Hopkins School of Medicine, Baltimore, USA

Correspondence: Dr Ben Sessa, AddAction, 35 The Boulevard, Weston-Super-Mare BS23 1PE, UK. Email: bensessa@gmail.com

First received 22 Nov 2013, final revision 10 Mar 2014, accepted 7 Oct 2014

\section{References}

1 James W. The Varieties of Religious Experience: 263. Penguin Classics, 1983 (first edition 1902) 
2 National Collaborating Centre for Mental Health. Alcohol-Use Disorders. Diagnosis, Assessment and Management of Harmful Drinking and Alcoho Dependence. NICE Clinical Guideline 115. British Psychological Society \& Royal College of Psychiatrists, 2011

3 Harkins C, Morleo M, Cook PA. Evaluation of the Use of Alcohol Treatment Requirements and Alcohol Activity Requirements for Offenders in Cheshire (http://www.cph.org.uk/wp-content/uploads/2012/08/evaluation-of-the-useof-alcohol-treatment-requirement-and-alcohol-activity-requirements-foroffenders-in-cheshire.pdf). Centre for Public Health \& Cheshire Probation, 2011.

4 Miller WR, Wilbourne PL. Mesa Grande: a methodological analysis of clinica trials of treatments for alcohol use disorders. Addiction 2002; 97: 265-77.

5 Home Office. The Government's Alcohol Strategy. Cm 8336. TSO (The Stationery Office), 2012.

6 Hickman M, Carnwath Z, Madden P, Farrell M, Rooney C, Ashcroft R, et al. Drug-related mortality and fatal overdose risk: pilot cohort study of heroin users recruited from specialist drug treatment sites in London. J Urban Health 2003; 80: 274-87.

7 Connock M, Juarez-Garcia A, Jowett S, Frew E, Liu Z, Taylor RJ, et al. Methadone and buprenorphine for the management of opioid dependence: a systematic review and economic evaluation. Health Technol Assess 2007 11: 1-171.

8 World Health Organization Regional Office for Europe. The European Tobacco Control Report 2007. WHO, 2007.
9 Reus VI, Smith BJ. Multimodal techniques for smoking cessation: a review of their efficacy and utilisation and clinical practice guidelines. Int J Clin Pract 2008; 62: 1753-68.

10 Dyck E. Psychedelic Psychiatry: LSD from Clinic to Campus. Johns Hopkins University Press, 2008.

11 Doblin R. Dr Leary's Concord Prison experiment: a 34-year follow-up study. Psychoactive Drugs 1998; 30: 419-26.

12 Hendricks PS, Clark CB, Johnson MW, Fontaine KR, Cropsey KL. Hallucinogen use predicts reduced recidivism among substance-involved offenders under community corrections supervision. J Psychopharmacol 2014; 28: 62-6.

13 MacLean KA, Johnson MW, Griffiths RR. Mystical experiences occasioned by the hallucinogen psilocybin lead to increases in the personality domain of openness. J Psychopharmacol 2011; 25: 1453-61.

$14 \mathrm{Halpern} \mathrm{JH}$. The use of hallucinogens in the treatment of addiction. Addiction Res 1996; 4: 177-89.

15 Bogenschutz MP, Pommy JM. Therapeutic mechanisms of classic hallucinogens in the treatment of addictions: from indirect evidence to testable hypotheses. Drug Test Anal 2012; 4: 543-55.

16 Krupitsky EM, Grinenko AY. Ketamine psychedelic therapy (KPT): a review of the results of ten years of research. J Psychoactive Drugs 1997; 29: 165-83.

17 Sessa B. Shaping the renaissance of psychedelic research. Lancet 2012; $\mathbf{3 8 0}$ : 200-1. 